\title{
Le recyclage de l'aluminium: Enjeux pour le Saguenay-Lac-Saint-Jean
}

\author{
Sylvain Gaudreault \\ député de Jonquière à l'Assemblée nationale ${ }^{1}$
}

Pourquoi aborder le recyclage de l'aluminium? Du point de vue du développement durable et quand on considère tout le cycle de vie du métal gris, l'aluminium recyclé devient un matériau très intéressant. À première vue, il semble évident qu'il existe là une filière extrêmement prometteuse pour une région tentée de s'y investir le moindrement, surtout si cette région prétend au titre de «Vallée de l'aluminium »...

\section{Le cycle de vie}

Dans une perspective de développement durable, il semble préférable de considérer la production industrielle en vertu du cycle de vie complet de la matière transformée. D'ailleurs, l'industrie de l'aluminium elle-même adopte ce point de vue d'un produit qui est «développement durable » dans la mesure où tout le cycle de vie de l'aluminium est pris en compte. Nous pouvons identifier sept étapes distinctes de ce cycle de vie : l'extraction de la bauxite, la production d'alumine, la production d'aluminium primaire, la transformation de l'aluminium, la fabrication de produits en aluminium, la phase d'utilisation et, enfin, le recyclage. Cette dernière étape conduit à refaire le cycle de vie à partir de la transformation de l'aluminium.

La refonte de l'aluminium usagé permet d'économiser jusqu'à $95 \%$ de l'énergie nécessaire à produire le métal primaire. $\mathrm{Au}$ Canada, recycler une tonne d'aluminium permet d'éviter que 2,8 tonnes de $\mathrm{C} 02$ ne se dispersent dans l'atmosphère. Recycler un
Nous savons que la production d'aluminium primaire est une activité «polluante». Ainsi, si nous recyclons le produit, nous contribuons au développement durable. En outre, quand l'aluminium est utilisé pour construire des véhicules plus légers, on réduit du coup la pollution de ces mêmes véhicules.

\section{Il semble évident qu'il existe là une filière extrêmement prometteuse pour une région tentée de s'y investir le moindrement}

kilogramme d'aluminium permet d'économiser environ $8 \mathrm{~kg}$ de bauxite, $4 \mathrm{~kg}$ de produits chimiques et 14 kilowatts/heure d'électricité. Si nous traduisons ces données en exemples concrets, nous pouvons affirmer que chaque canette d'aluminium recyclée permet d'économiser en énergie l'équivalent d'une ampoule de 100 watts allumée pendant 3,5 heures. On évalue qu'il faut environ 670 canettes d'aluminium pour fabriquer un vélo!

Du point de vue financier, le recyclage de l'aluminium « s'autofinance » grâce à la grande valeur de ce genre de rebuts. Une tonne de canettes d'aluminium récupéré a une valeur approximative pouvant dépasser $2000 \$$. Au niveau mondial, les rebuts d'aluminium sont considérés comme des matières premières.

La refonte de l'aluminium usagé permet d'économiser jusqu'à $95 \%$ de l'énergie nécessaire à produire le métal primaire 
Comment le Saguenay-Lac-Saint-Jean se classe-t-il dans le cycle de vie de l'aluminium? La région est très présente dans la production d'alumine et dans la production d'aluminium primaire. Malgré des hauts et des bas, la présence du Saguenay-Lac-Saint-Jean se développe aussi dans la transformation et dans la fabrication de produits. Dans ce contexte, le

\section{Un potentiel inexploité}

L'aluminium recyclé est une industrie en croissance. La part de l'aluminium recyclé dans la consommation totale d'aluminium est passée de moins de $20 \%$ dans les années 1950 à environ $33 \%$ en 2006. L'International Aluminium Institute (IAI) estime que le ratio d'aluminium recyclé dans la production totale passera à $40 \%$ en 2020. L'industrie de l'aluminium recyclé a triplé sa production de 5 millions de tonnes en 1980 à 16 millions de tonnes en 2006. Pendant cette même période et à titre comparatif, l'utilisation de métal primaire est passée de 15 à 30 millions de tonnes. Il y a là des occasions d'affaires à saisir!

Le plein potentiel de l'aluminium recyclé apparaît encore inexploité. En effet, environ $75 \%$ de l'aluminium utilisé dans les automobiles est récupéré et recyclé. Pour les emballages, le taux de récupération est plus bas, soit autour de $30 \%$ (selon l'Industrie de l'aluminium pour le développement durable). Pour les canettes, le taux de recyclage varie beaucoup d'un pays à l'autre : $90 \%$ en Suisse, $78 \%$ au Brésil et environ $50 \%$ aux ÉtatsUnis. Au Québec, $68 \%$ des contenants à remplissage unique en aluminium sont recyclés selon Recyc-Québec (2007). Autrement dit, même près de chez nous, il reste encore une bonne quantité d'aluminium qui n'est pas recyclé! Sur la planète, plus de 50 milliards de canettes ne seraient pas récupérées recyclage apparaît comme le maillon faible. Il se fait un peu de recyclage des rebuts de fabrication des alumineries (par exemple chez Alumitherm Inc. et chez Scepter Aluminium Company), mais le chantier du recyclage des produits en aluminium reste entier dans la région.

chaque année, ce qui représente près de 800 000 tonnes d'aluminium, soit l'équivalent de près de 17 millions de barils de pétrole brut ou encore à la quantité d'électricité nécessaire pour alimenter 2,8 millions de familles américaines pendant un an!

Le facteur énergétique pourrait augmenter l'avantage lié au recyclage. En effet, on peut s'attendre à ce que l'augmentation des coûts d'énergie résultant $\mathrm{du}$ 《 choc pétrolier » accroisse la demande pour le métal à recycler, puisque l'aluminium recyclé requiert beaucoup moins d'énergie à produire.

\section{Le plein potentiel de l'aluminium recyclé apparaît encore inexploité}

Par contre, le recyclage d'aluminium comporte aussi ses vices dont il est important de tenir compte. D'abord, le volume de rebuts se trouve là où les densités de population sont les plus grandes, ce qui rend le Saguenay-LacSaint-Jean moins attrayant pour cette industrie. Ensuite, tous les produits confectionnés en aluminium ne sont pas composés des mêmes alliages. C'est pourquoi un tri pointu par famille d'alliages et une bonne qualité du prétraitement (ex. : nettoyage) augmentent la valeur des rebuts d'aluminium et facilitent leur réutilisation. 


\section{Et le Saguenay-Lac-Saint-Jean?}

Dans ce contexte, la question est de savoir s'il y a des occasions d'affaires à saisir pour le Saguenay-Lac-Saint-Jean. Nous croyons qu'il serait utopique d'espérer transporter dans la Vallée de l'aluminium toutes les canettes de l'Amérique du Nord. C'est pourquoi nous pensons qu'il vaut mieux viser des marchés de niche, notamment dans le savoir-faire et les procédés (par exemple les méthodes pour améliorer le tri ou le prétraitement, la recherche dans la conception et la fabrication facilitant le recyclage, etc.).

À notre avis, face à l'énorme potentiel que représente le recyclage de l'aluminium, nous croyons que les pistes à explorer pour le Saguenay-Lac-Saint-Jean sont les suivantes :

- Identifier des applications potentielles;

- Évaluer les occasions au cas par cas avec une approche d'affaires;
- Encourager la vigie, la recherche et le maillage;

- Continuer à soutenir les centres de recherche, la Vallée de l'aluminium et la masse critique d'équipementiers et d'industriels présents au Saguenay-LacSaint-Jean, et ce, dans l'optique d'accroître le potentiel du recyclage.

En terminant, une chose nous apparaît sûre : le recyclage de l'aluminium est un enjeu de développement durable et s'inscrit dans l'avenir, tant du point de vue de son potentiel de croissance que des occasions d'affaires qu'il représente. À ce titre, pour une région comme le Saguenay-Lac-Saint-Jean qui est déjà bien présente dans le cycle de vie de l'aluminium, l'industrie du recyclage du métal gris mérite d'être examinée avec soin.

\section{Notes et références}

${ }^{1}$ L'auteur tient à remercier grandement madame Denise Turcotte, conseillère politique à son bureau de comté, pour la recherche effectuée dans ce dossier.

Alcan, Rapport sur la durabilité d'entreprise, 2002 :

http://www.publications.alcan.com/sustainability/fr/steps/enviro_03.html

Alcan, Alcan est en tête des efforts d'économie d'énergie grâce à un taux de recyclage record, 2001 : AllBusiness :

http://www.alcan.com/web/publishing.nsf/Content/Alcan+leads+energy+savings+through+record+recycling+efforts_FR

http://www.allbusiness.com/primary-metal-manufacturing/alumina-aluminum/232827-1.html

Association de l'aluminium du Canada, Bâtir un futur durable pour l'aluminium recyclé :

http://www.aac.aluminium.qc.ca/frameset/pdf/Futur_durable.pdf

Earth911, Facts about Aluminium Recycling :

http://www.earth911.org/master.asp?s=lib\&a=aluminum/facts.asp

GENTAZ, Claude, Aluminium, développement durable et énergie, Centre Universitaire des Problèmes de l'Énergie, Université de Genève et

Transtec :

http://ecolu-info.unige.ch/colloques/SMIA03/archives/SMIA03Gentaz.pdf

Industrie de l'aluminium pour le développement durable :

http://www.aluminium-futur.com/cycle_vie/cycle7.htm

http://www.aluminium-futur.com/fiches/recyclage.htm

International Aluminium Institute :

http://www.world-aluminum.org/iai/stats/formServer.asp?form=1

http://www.world-aluminum.org/iai/stats/formServer.asp?form $=14$

http://www.world-aluminium.org/Sustainability/Recycling

Radio-Canada, «L'entreprise de recyclage d'aluminium Alsa ferme temporairement une de ses usines », 22 juillet 2005 : http://www.radio-canada.ca/regions/estrie/nouvelles/200507/22/009-aluminium.shtml

Recyc-Québec :

http://www.recyc-quebec.gouv.qc.ca/prorecyc/saviezvous/consignes/aluminium/index.htm

- $\quad$ http://www.recyc-quebec.gouv.qc.ca/client/fr/rubriques/Nouvelles.asp?id=439 


\section{PUBLICITÉ}

Revue Internationale PME 